\title{
FIBONACCI-HORNER DECOMPOSITION OF THE MATRIX EXPONENTIAL AND THE FUNDAMENTAL SYSTEM OF SOLUTIONS*
}

\author{
R. BEN TAHER ${ }^{\dagger}$, M. MOULINE ${ }^{\ddagger}$, AND M. RACHIDI $^{\$}$
}

\begin{abstract}
This paper concerns the Fibonacci-Horner decomposition of the matrix powers $A^{n}$ and the matrix exponential $e^{t A}(A \in M(r ; \mathbb{C}), t \in \mathbb{R})$, which is derived from the combinatorial properties of the generalized Fibonacci sequences in the algebra of square matrices. More precisely, $e^{t A}$ is expressed in a natural way in the so-called Fibonacci-Horner basis with the aid of the dynamical solution of the associated ordinary differential equation. Two simple processes for computing the dynamical solution and the fundamental system of solutions are given. The connection to VerdeStar's approach is discussed. Moreover, an extension to the computation of $f(A)$, where $f$ is an analytic function is initiated. Finally, some illustrative examples are presented.
\end{abstract}

Key words. Matrix powers, Matrix exponential, Dynamical solution, Generalized Fibonacci sequence, Fibonacci-Horner decomposition, Matrix functions.

AMS subject classifications. 15A18, 15A99.

1. Introduction. It is well known that the computation of $e^{t A}$, where $A \in$ $M(r ; \mathbb{C})$ (the algebra of square $r \times r$ matrices) plays a central role in many fields of mathematics and applied sciences. There is a large variety of methods for computing the exponential of a matrix and the differential equation method is among them; see $[4,6,7,9,12,13]$ and references therein. As a consequence, diverse decompositions of $e^{t A}$ have been considered in the literature, associated with various kinds of bases for the vector space of polynomials; see, e.g., $[4,6,7,9,12,13,14]$. An approach for the decomposition of $e^{t A}$ related to the combinatorial expression of generalized Fibonacci sequences (see $[8,10]$ ) is studied in the recent papers $[1,2,11]$. Indeed, many important results in $[1,2,11]$ are derived from combinatorial expressions of these scalar recursive sequences that have been extended to linear recursive relations in the algebra of square matrices.

In this paper we provide two elementary methods for computing the exponential $e^{t A}(A \in M(r ; \mathbb{C}), t \in \mathbb{R})$ in the Fibonacci-Horner basis and give their applications in some simple situations. Our approach is based on the connection between the linear recurrence relations in $M(r ; \mathbb{C})$ and a combinatorial method for computing the powers $A^{n}$ and $e^{t A}$ (see [1, 2, 11]). More precisely, the aforementioned decomposition of $e^{t A}$ is derived from two simple practical processes for computing the dynamical solution of a specific scalar $r$-th order homogeneous linear differential equation. Moreover, the

\footnotetext{
${ }^{*}$ Received by the editors 2 November 2005. Accepted for publication 16 April 2006. Handling Editor: Richard A. Brualdi.

†Département de Mathématiques et Informatique, Faculté des Sciences, Université Moulay Ismail, B.P. 4010, Beni M’hamed, Méknés - Morocco (bentaher@fsmek.ac.ma).

${ }^{\ddagger}$ Département de Mathématiques et Informatique, Faculté des Sciences, Université Mohammed V - B.P. 1014, Rabat - Morocco (mouline@fsr.ac.ma).

$\S$ Mathematics Section, LEGT-F. Arago - Académie de Reims. 1, Rue F. Arago, 51100 Reims France (mu.rachidi@wanadoo.fr).
} 
usual polynomial decomposition of $e^{t A}$ can be recovered. Finally, an application of the preceding methods to the computation of $f(A)$, where $f$ is an analytic function, is also initiated.

This paper is organized as follows. In Section 2, using a standard computation, we give the basic Fibonacci-Horner basis, as well as the relationship between the matrix exponential and the system of fundamental solutions of an associated scalar linear differential equation of order $r$. We also state our main problem of computing explicitly the dynamical solution and the fundamental system of solutions of this differential equation. In Section 3, we illustrate our first method, called elimination process, providing the dynamical solution of the preceding differential equation. The decomposition of $e^{t A}$ in some important situations is also studied. In Section 4, we give a functional process which permits us to establish an explicit expression of the dynamical solution and obtain the Fibonacci-Horner decomposition of $e^{t A}$. Also, a connection between our approach and the one by Verde-Star is established. In Section 5, we improve the Fibonacci-Horner decomposition of $f(A)$, when $f$ is an analytic function. Finally, Section 6 is devoted to some illustrative examples.

2. Fibonacci-Horner decomposition of the matrix exponential and dynamical solution. Let $A \in M(r ; \mathbb{C})$ and let $P(z)=z^{r}-a_{0} z^{r-1}-\cdots-a_{r-1}$ be its characteristic polynomial. Following the Cayley-Hamilton Theorem we have $P(A)=\Theta_{r}$ (the zero $r \times r$ matrix). Therefore, the sequence of matrices $\left\{A^{n}\right\}_{n \geq 0}$ is an $r$-generalized Fibonacci sequence in $M(r ; \mathbb{C})$, whose coefficients and initial conditions are $a_{0}, \cdots, a_{r-1}$ and $A^{0}=I_{r}$ (the identity matrix), $A, \cdots, A^{r-1}$, respectively. When $A$ is invertible, we consider for reasons of convenience the $r$-generalized Fibonacci sequence $\left\{A^{n}\right\}_{n \geq-r+1}$, whose coefficients and initial conditions are $a_{0}, \cdots, a_{r-1}$ and $A^{-r+1}, \cdots, A^{-1}, A^{0}=I_{r}$, respectively. Following results of $[1,10,11]$, we have

$$
A^{n}=u_{n} A_{0}+u_{n-1} A_{1}+\cdots+u_{n-r+1} A_{r-1}, \text { for every } n \geq r,
$$

such that

$$
A_{0}=I_{r} ; A_{i}=A^{i}-a_{0} A^{i-1}-\cdots-a_{i-1} I_{r}, \text { for every } i=1, \cdots, r-1
$$

and the sequence $\left\{u_{n}\right\}_{n \geq-r+1}$ defined by,

$$
u_{n}=\sum_{k_{0}+2 k_{1}+\cdots+r k_{r-1}=n} \frac{\left(k_{0}+k_{1}+\cdots+k_{r-1}\right) !}{k_{0} ! k_{1} ! \cdots k_{r-1} !} a_{0}^{k_{0}} a_{1}^{k_{1}} \cdots a_{r-1}^{k_{r-1}}
$$

for every $n \geq 1$, with $u_{0}=1$ and $u_{n}=0$ for $-r+1 \leq n \leq-1$. It was established in $[8,10]$ that the sequence $\left\{u_{n}\right\}_{n \geq-r+1}$ satisfies the following linear recurrence relation:

$$
u_{n+1}=a_{0} u_{n}+a_{1} u_{n-1}+\cdots+a_{r-1} u_{n-r+1} \text {, for every } n \geq 0 \text {. }
$$

In other words, $\left\{u_{n}\right\}_{n \geq-r+1}$ is an $r$-generalized Fibonacci sequence.

In fact, a simple induction shows that the decomposition (2.1) of the powers $A^{n}$ $(n \geq r)$ is still valid for every $r \times r$ complex matrix $A$ (invertible or not), with the $A_{j}$ $(0 \leq j \leq r-1)$ given by $(2.2)$ and the sequence $\left\{u_{n}\right\}_{n \geq-r+1}$ by $(2.3)$. 
The set of matrices $\left\{A_{0}, A_{1}, \cdots, A_{r-1}\right\}$ is called the Fibonacci-Horner basis of the power decomposition (2.1) of $A$. Indeed, this basis is derived from the Fibonacci combinatorial process and we show that each matrix $A_{j}(0 \leq j \leq r-1)$ given by $(2.2)$ satisfies $A_{j}=h_{j}(A)$, where $h_{0}(z)=1, h_{1}(z)=z-a_{0}, \cdots, h_{j}(z)=z h_{j-1}(z)-a_{j-1}$, $\cdots, h_{r-1}(z)=z h_{r-2}(z)-a_{j-2}$ and $h_{r}(z)=z h_{r-1}(z)-a_{r-1}=P(z)$ are the Horner polynomials associated with the characteristic polynomial $P(z)$.

For the computation of $e^{t A}$ we consider the expression $e^{t A}=\sum_{n \geq 0} \frac{t^{n}}{n !} A^{n}$. A straightforward computation using (2.1)-(2.3) allows us to derive the following result.

Proposition 2.1. Let $A \in M(r ; \mathbb{C})$ with characteristic polynomial $P(z)=$ $z^{r}-a_{0} z^{r-1}-\cdots-a_{r-1}$. Then,

$$
e^{t A}=\varphi^{(r-1)}(t) A_{0}+\varphi^{(r-2)}(t) A_{1}+\cdots+\varphi(t) A_{r-1}
$$

where $\varphi^{(k)}(t)$ is the $k$-th derivative of function,

$$
\varphi(t)=\sum_{n=0}^{+\infty} u_{n} \frac{t^{n+r-1}}{(n+r-1) !}
$$

Expression (2.4) shows that $\varphi(t)$ satisfies the following ordinary differential equation $y^{(r)}(t)=a_{0} y^{(r-1)}(t)+a_{1} y^{(r-2)}(t)+\cdots+a_{r-1} y(t)$. Moreover, using (2.6), we establish easily that the $k$-th derivative of the function $\varphi(t)$ satisfies $\varphi^{(k)}(0)=0$ for $k=0,1, \cdots, r-2$ and $\varphi^{(r-1)}(0)=1$. Hence, the function $\varphi(t)$ given by $(2.6)$ is nothing else but the dynamical solution of the preceding differential equations (see $[7,13])$. Also (2.5) shows that the coefficients of $e^{t A}$ in the Fibonacci-Horner basis are the elements of the fundamental system of solutions $\left\{\varphi(t), \varphi^{\prime}(t), \cdots, \varphi^{(r-2)}(t), \varphi^{(r-1)}(t)\right\}$ of the preceding differential equation.

By Proposition 2.1 the decomposition of $e^{t A}$ in the Fibonacci-Horner basis, depends on the knowledge of the dynamical solution $\varphi(t)$ given by $(2.6)$. The goal of the next section is to study the question of computing explicit formulas for this function.

REMARK 2.2. Expressions (9)-(10) of [1] give the closed relation between the Fibonacci decomposition and the polynomial decomposition of the powers and the exponential of matrices. Expressions (2.1)-(2.2) give $A^{n}=\rho_{0}(n) I_{r}+\rho_{1}(n) A+\cdots+$ $\rho_{r-1}(n) A^{r-1}$, where $\rho_{k}(n)=u_{n-k-1}-\sum_{j=1}^{k} a_{r-k+j-1} u_{n-j}$. Therefore, (2.5) implies that we have the polynomial decomposition $e^{t A}=\sum_{j=0}^{r-1} \Omega_{j}(t) A^{j}$, where $\Omega_{k}(t)=$ $\frac{t^{k}}{k !}+\sum_{n \geq r} \frac{t^{n}}{n !} \rho_{k}(n)($ see $[1])$.

REMARK 2.3. In [13] improved expressions for $A^{n}$ and $e^{t A}$ are obtained with the aid of the dynamical solution. In the latter case, this is done in various forms, using a specific technique of the theory of divided differences. In Verde-Star's approach Horner polynomials play an important role. Moreover, this approach is also considered in [14] for the study of functions of matrices in connection with partial fraction decompositions (see [5]). We shall discuss this aspect in Subsection 4.2 below. 
REMARK 2.4. Regarding expression (2.6) of the dynamical solution and its derivatives, knowledge of the eigenvalues of the matrix $A$ is not necessary. ¿From a computational point of view it seems more convenient to consider (2.6). However, the problem with this expression is that the coefficients $u_{n}$ may grow fast and somewhat irregularly. This occurs when approximations in (2.6) are considered. For this problem it seems natural to take into account properties of the asymptotic behavior of $r$-generalized Fibonacci sequences that have been studied in the literature (see, e.g., [3]). Indeed, this may help to control asymptotically the growth and the irregularity of $u_{n}$ for large $n$, and to study approximations of (2.6).

3. Fibonacci-Horner decomposition of $e^{t A}$ : elimination process. Let $A \in M(r ; \mathbb{C})$ and suppose that $\lambda_{1}, \lambda_{2}, \cdots, \lambda_{p}$ are the distinct roots of its characteristic polynomial $P(z)=z^{r}-a_{0} z^{r-1}-\cdots-a_{r-1}$, having multiplicities $m_{1}, \cdots, m_{p}$, respectively. It is well known that the dynamical solution $\varphi(t)$ given by $(2.6)$ can be expressed in the following form:

$$
\varphi(t)=R_{1}(t) e^{t \lambda_{1}}+R_{2}(t) e^{t \lambda_{2}}+\cdots+R_{p}(t) e^{t \lambda_{p}},
$$

where $R_{j}(t)(1 \leq j \leq p)$ are polynomial functions of degree $m_{j}-1$. More precisely, the polynomials $R_{j}(t)(1 \leq j \leq p)$ are derived from the initial conditions

$$
\varphi(0)=\varphi^{\prime}(0)=\cdots=\varphi^{(r-2)}(0)=0, \text { and } \varphi^{(r-1)}(0)=1 .
$$

For a matrix $A$ of large order $r$, computing the polynomials $R_{j}(t)(1 \leq j \leq p)$ by (3.2) is not a very simple task in general. Meanwhile, recently Verde-Star's theory permits to obtain the polynomials $R_{j}(t)(1 \leq j \leq s)$ using the method of divided difference (see [13]). In Subsection 4.2 we bring focus to this observation.

The aim of this section is to disassociate the value of the polynomials $R_{j}(t)$ $(1 \leq j \leq p)$ from the linear systems that we construct, using only a direct computation and without resorting to existing techniques in the literature. Thus, we derive the explicit Fibonacci-Horner decomposition of $e^{t A}$ with the aid of (2.5)-(2.6) and (3.2). For reasons of clarity, we shall first illustrate our method in the three cases $p=1,2$ and 3 .

Suppose that the characteristic polynomial $P(z)=z^{r}-a_{0} z^{r-1}-\cdots-a_{r-1}$ of the matrix $A$ has a unique root $\lambda$; that is, $P(z)=(z-\lambda)^{r}$. Therefore, expression (3.1) of the dynamical solution can be written as $\varphi(t)=R(t) e^{\lambda t}$, where $R(t)$ is a polynomial of degree $r-1$. If we consider the Taylor expansion of $R(t)$ we have $R(t)=\sum_{k=0}^{r-1} \frac{R^{(k)}(0)}{k !} t^{k}=\varphi(t) e^{-\lambda t}$. The initial conditions (3.2) imply that $R^{(k)}(0)=0$ for $0 \leq k \leq r-2$ and $R^{(r-1)}(0)=1$. Hence, we have the following result.

Proposition 3.1. Let $A \in M(r ; \mathbb{C})$ with characteristic polynomial $P(z)=$ $(z-\lambda)^{r}$. Then, $e^{t A}=\varphi^{(r-1)}(t) A_{0}+\varphi^{(r-2)}(t) A_{1}+\cdots+\varphi(t) A_{r-1}$, where $\varphi^{(k)}(t)$ is the $k$-th derivative of the function $\varphi(t)=\frac{t^{r-1}}{(r-1) !} e^{t \lambda}$. 
Suppose that the characteristic polynomial $P(z)=z^{r}-a_{0} z^{r-1}-\cdots-a_{r-1}$ of the matrix $A$ has two distinct roots $\lambda_{1}$ and $\lambda_{2}$, of multiplicities $m_{1}$ and $m_{2}$, respectively, where $m_{1}+m_{2}=r$. Hence, we have $P(z)=\left(z-\lambda_{1}\right)^{m_{1}}\left(z-\lambda_{2}\right)^{m_{2}}$ and expression (3.1) of the dynamical solution can be written as $\varphi(t)=R_{1}(t) e^{\lambda_{1} t}+R_{2}(t) e^{\lambda_{2} t}$, where $R_{j}(t)(j=1,2)$ is a polynomial of degree $m_{j}-1$. The main task here is to determine the explicit formulas of the polynomials $R_{j}(t)(j=1,2)$. Starting from the Taylor expansions $R_{j}(t)=\sum_{k=0}^{m_{j}-1} \frac{R_{j}^{(k)}(0)}{k !} t^{k}$ of $R_{j}(t)(j=1,2)$, we determine first the polynomial $R_{1}(t)$. Since $R_{2}(t)$ is of degree $m_{2}-1$ and $\varphi(t) e^{-\lambda_{2} t}=R_{1}(t) e^{\left(\lambda_{1}-\lambda_{2}\right) t}+R_{2}(t)$, we can eliminate $R_{2}(t)$ after the derivative of order $m_{2}$ of both sides of this later expression. Indeed, we have

$$
\psi(t)=\varphi_{1}(t) e^{-\lambda_{2} t}=\sum_{l=0}^{m_{2}}\left({ }_{m_{2}}^{l}\right) R_{1}^{(l)}(t)\left(\lambda_{1}-\lambda_{2}\right)^{m_{2}-l},
$$

where $\left(\begin{array}{l}j \\ n\end{array}\right)=\frac{n !}{j !(n-j) !}$ and $\varphi_{1}(t)=\sum_{s=0}^{m_{2}}\left(\begin{array}{c}s \\ m_{2}\end{array}\right)\left(-\lambda_{2}\right)^{m_{2}-s} \varphi^{(s)}(t)$. If we set $C_{l}=$ $\left(\begin{array}{l}l \\ m_{2}\end{array}\right)\left(\lambda_{1}-\lambda_{2}\right)^{m_{2}-l}$, we have $\psi^{(k)}(t)=\sum_{l=0}^{m_{2}} C_{l} R_{1}^{(l+k)}(t)$, with $0 \leq l+k \leq m_{1}-1$. Since $\varphi^{(k)}(0)=0$ for $0 \leq k \leq r-2$ and $\varphi^{(r-1)}(0)=1$, we derive that $\varphi_{1}^{(k)}(0)=0$ for $0 \leq k \leq m_{1}-2$ and $\varphi_{1}^{\left(m_{1}-1\right)}(0)=1$. Hence, for $k=m_{1}-1$ we have $\psi^{\left(m_{1}-1\right)}(0)=1$ and $\psi^{(k)}(0)=0$ for $k=0, \cdots, m_{1}-2$. These equations imply that the Taylor coefficients $R_{1}^{(k)}(0)\left(0 \leq k \leq m_{1}-1\right)$ of the polynomial $R_{1}(t)$ satisfy the following system of linear equations:

$$
C_{0} R_{1}^{\left(m_{1}-1\right)}(0)=1 \text { and } \sum_{j=0}^{s-1} C_{j} R_{1}^{\left(m_{1}-s+j\right)}(0)=0 \text { for } 2 \leq s \leq m_{1}-1 \text {. }
$$

The same argumentation shows that the Taylor coefficients of the polynomial $R_{2}(t)$ satisfy the following system of linear equations:

$$
K_{0} R_{2}^{\left(m_{2}-1\right)}(0)=1 \text { and } \sum_{j=0}^{s-1} K_{j} R_{2}^{\left(m_{2}-s+j\right)}(0)=0 \text { for } 2 \leq s \leq m_{2},
$$

where $K_{l}=\left(\begin{array}{l}l \\ m_{2}\end{array}\right)\left(\lambda_{2}-\lambda_{1}\right)^{m_{2}-l}$. Since $\lambda_{1} \neq \lambda_{2}$, a simple iterative process shows that each one of the two systems (3.3) and (3.4) has a unique solution.

Proposition 3.2. Let $A \in M(r ; \mathbb{C})$ with characteristic polynomial $P(z)=$ $\left(z-\lambda_{1}\right)^{m_{1}}\left(z-\lambda_{2}\right)^{m_{2}}\left(m_{1}+m_{2}=r\right)$. Then, $e^{t A}=\varphi^{(r-1)}(t) A_{0}+\varphi^{(r-2)}(t) A_{1}+\cdots+$ $\varphi(t) A_{r-1}$, where $\varphi(t)=R_{1}(t) e^{t \lambda_{1}}+R_{2}(t) e^{t \lambda_{2}}$ is the associated dynamical solution; $R_{1}(t), R_{2}(t)$ are polynomials of degree $m_{1}-1, m_{2}-1$, respectively, whose Taylor coefficients $R_{j}^{(k)}(0)\left(j=1,2\right.$ and $\left.0 \leq k \leq m_{j}-1\right)$ are solutions of the two elementary linear systems of equations (3.3)-(3.4).

The preceding method can be extended to the case of distinct roots $\lambda_{1}, \cdots, \lambda_{p}$ $(p \geq 3)$ of $P(z)$. For reason of clarity, let us suppose that $P(z)=z^{r}-a_{0} z^{r-1}-$ 
$\cdots-a_{r-1}$ has three distinct roots $\lambda_{1}, \lambda_{2}$ and $\lambda_{3}$ of multiplicities $m_{1}, m_{2}$ and $m_{3}$, respectively, where $m_{1}+m_{2}+m_{3}=r$; that is $P(z)=\left(z-\lambda_{1}\right)^{m_{1}}\left(z-\lambda_{2}\right)^{m_{2}}\left(z-\lambda_{3}\right)^{m_{3}}$. Therefore, (3.1) can be written as $\varphi(t)=R_{1}(t) e^{\lambda_{1} t}+R_{2}(t) e^{\lambda_{2} t}+R_{3}(t) e^{\lambda_{3} t}$, where $R_{j}(t)(j=1,2,3)$ is a polynomial of degree $m_{j}-1$. The main task here is to determine the explicit expressions of each polynomial $R_{j}(t)(j=1,2,3)$. As in the preceding case, we are going to exhibit the polynomial $R_{1}(t)$. To this aim, we consider the Taylor expansions $R_{j}(t)=\sum_{k=0}^{m_{j}-1} \frac{R_{j}^{(k)}(0)}{k !} t^{k}$ of $R_{j}(t)(j=1,2,3)$. Since $R_{3}(t)$ is of degree $m_{3}-1$ and $\varphi(t) e^{-\lambda_{3} t}=R_{1}(t) e^{\left(\lambda_{1}-\lambda_{3}\right) t}+R_{2}(t) e^{\left(\lambda_{2}-\lambda_{3}\right) t}+R_{3}(t)$, we derive that

$$
\varphi_{3}(t) e^{-\lambda_{3} t}=\left(\sum_{j=0}^{m_{3}}\left({ }_{m_{3}}^{j}\right) R_{1}^{(j)}(t)\left(\lambda_{1}-\lambda_{3}\right)^{m_{3}-j}\right) e^{\left(\lambda_{1}-\lambda_{3}\right) t}+R_{2,3}(t) e^{\left(\lambda_{2}-\lambda_{3}\right)}
$$

where $\varphi_{3}(t)=\sum_{j=0}^{m_{3}}\left({ }_{m_{3}}^{j}\right)\left(-\lambda_{3}\right)^{m_{3}-j} \varphi^{(j)}(t)$ and $R_{2,3}(t)$ is a polynomial of degree $m_{2}-$ 1. We show that the polynomial $R_{3}(t)$ is eliminated after $m_{3}$ derivations of both sides of (3.5). In the same way, after some simplifications and $m_{2}$ derivations of both sides of Expression (3.5), we also eliminate the polynomial $R_{2,3}(t)$. More precisely, we have

$$
\left.\frac{d^{m_{2}}}{d t^{m_{2}}}\left[\varphi_{3}(t)\right) e^{-\lambda_{2} t}\right]=\left(\sum_{0 \leq i \leq m_{2} ; 0 \leq j \leq m_{3}} C_{i, j} R_{1}^{(i+j)}(t)\right) e^{\left(\lambda_{1}-\lambda_{2}\right) t},
$$

where $C_{i, j}=\left(\begin{array}{l}i \\ m_{2}\end{array}\right)\left(\begin{array}{l}j \\ m_{3}\end{array}\right)\left(\lambda_{1}-\lambda_{2}\right)^{m_{2}-i}\left(\lambda_{1}-\lambda_{3}\right)^{m_{3}-j}$. On the other hand, the left side of (3.6) can be written as

$$
\frac{d^{m_{2}}}{d t^{m_{2}}}\left[\varphi_{3}(t) e^{-\lambda_{3} t}\right]=\varphi_{2}(t) e^{-\lambda_{2} t}
$$

where $\varphi_{2}(t)=\sum_{j=0}^{m_{2}}\left({ }_{m_{2}}^{j}\right)\left(-\lambda_{2}\right)^{m_{2}-j} \varphi_{3}^{(j)}(t)$. Therefore, we have

$$
\Psi(t)=\varphi_{2}(t) e^{-\lambda_{1} t}=\sum_{0 \leq i \leq m_{2} ; 0 \leq j \leq m_{3}} C_{i, j} R_{1}^{(i+j)}(t),
$$

where $C_{i, j}=\left(\begin{array}{l}i \\ m_{2}\end{array}\right)\left(\begin{array}{l}j \\ m_{3}\end{array}\right)\left(\lambda_{1}-\lambda_{2}\right)^{m_{2}-i}\left(\lambda_{1}-\lambda_{3}\right)^{m_{3}-j}$. More precisely, (3.7) can be written as

$$
\Psi(t)=\sum_{s=0}^{m_{2}+m_{3}} C_{s} R_{1}^{(s)}(t)
$$

where

$$
C_{s}=\sum_{i+j=s ; 0 \leq i \leq m_{2} ; 0 \leq j \leq m_{3}}\left(\begin{array}{l}
i \\
m_{2}
\end{array}\right)\left(\begin{array}{l}
j \\
m_{3}
\end{array}\right)\left(\lambda_{1}-\lambda_{2}\right)^{m_{2}-i}\left(\lambda_{1}-\lambda_{3}\right)^{m_{3}-j} .
$$


Since $\varphi(t)$ is the dynamical solution given by $(2.6)$, we have $\varphi^{(k)}(0)=0$ for $0 \leq k \leq r-2$ and $\varphi^{(r-1)}(0)=1$. Therefore, (3.5) shows that $\varphi_{3}^{(k)}(0)=0$ for $0 \leq k \leq m_{1}+m_{2}-2$ and $\varphi_{3}^{\left(m_{1}+m_{2}-1\right)}(0)=1$. Hence, (3.7) implies that $\Psi^{(k)}(0)=0$, for $0 \leq k \leq m_{1}-2$ and $\Psi^{\left(m_{1}-1\right)}(0)=1$. Since

$$
\Psi^{(k)}(t)=\sum_{s=0}^{m_{2}+m_{3}} C_{s} R_{1}^{(s+k)}(t)
$$

we deduce that for $k=m_{1}-1, m_{1}-2, \cdots, 2,1,0$, respectively, the Taylor coefficients $R_{1}^{(k)}(0)\left(0 \leq k \leq m_{1}-1\right)$ of the polynomial $R_{1}(t)$ satisfy the following system of equations:

$$
C_{0} R_{1}^{\left(m_{1}-1\right)}(0)=1 \text { and } \sum_{j=0}^{s-1} C_{j} R_{1}^{\left(m_{1}-s+j\right)}(0)=0 \text { for } 2 \leq s \leq m_{1},
$$

where the $C_{j}$ are given by Expression (3.9). In the same way, the computation of the Taylor coefficients $R_{p}^{(k)}(0)$ of the polynomial $R_{p}(t)(p=2,3)$, are derived from the following systems of equations:

$$
D_{0} R_{2}^{\left(m_{2}-1\right)}(0)=1, \sum_{j=0}^{s-1} D_{j} R_{2}^{\left(m_{2}-s+j\right)}(0)=0 \text { for } 2 \leq s \leq m_{2}
$$

and

$$
E_{0} R_{3}^{\left(m_{3}-1\right)}(0)=1, \sum_{j=0}^{s-1} E_{j} R_{3}^{\left(m_{3}-s+j\right)}(0)=0 \text { for } 2 \leq s \leq m_{3} .
$$

Therefore, for $p=3$ the exponential $e^{t A}$ is given as follows.

Proposition 3.3. Let $A \in M(r ; \mathbb{C})$ with characteristic polynomial $P(z)=$ $\left(z-\lambda_{1}\right)^{m_{1}}\left(z-\lambda_{2}\right)^{m_{2}}\left(z-\lambda_{3}\right)^{m_{3}}\left(m_{1}+m_{2}+m_{3}=r\right)$. Then, $e^{t A}=\varphi^{(r-1)}(t) A_{0}+$ $\varphi^{(r-2)}(t) A_{1}+\cdots+\varphi(t) A_{r-1}$, where $\varphi(t)=R_{1}(t) e^{t \lambda_{1}}+R_{2}(t) e^{t \lambda_{2}}+R_{3}(t) e^{t \lambda_{3}}$ is the associated dynamical solution such that $R_{1}(t), R_{2}(t), R_{3}(t)$ are polynomials of degree $m_{1}-1, m_{2}-1, m_{3}-1$, respectively, whose Taylor coefficients $R_{j}^{(k)}(0)(j=12,3$, $\left.0 \leq k \leq m_{j}-1\right)$ are solutions of the three elementary linear system of equations (3.10), (3.11) and (3.12).

Following the same method, the process of elimination given in Propositions 3.1, 3.2 and 3.3 can be extended recursively to the general case. Indeed, we have the following general result.

Theorem 3.4. Let $A \in M(r ; \mathbb{C})(r \geq 2)$ with characteristic polynomial $P(z)=$ $\prod_{j=1}^{p}\left(z-\lambda_{j}\right)^{m_{j}}$, where $p \geq 3, m_{1}+\cdots+m_{p}=r$ and $\lambda_{i} \neq \lambda_{j}$ for $i \neq j$. For every $j$ 
$(1 \leq j \leq p)$ set

$$
C_{l}^{[j]}=\sum_{\sum_{1 \leq s \neq j \leq p} k_{s}=l}\left[\prod_{s=1, s \neq j}^{p}\left(\begin{array}{l}
k_{s} \\
m_{s}
\end{array}\right)\left(\lambda_{j}-\lambda_{s}\right)^{m_{s}-k_{s}}\right] .
$$

Then, $e^{t A}=\varphi^{(r-1)}(t) A_{0}+\varphi^{(r-2)}(t) A_{1}+\cdots+\varphi(t) A_{r-1}$, where $\varphi(t)=\sum_{j=1}^{p} R_{j}(t) e^{\lambda_{j} t}$ is the dynamical solution whose associated polynomials $R_{j}(t)(0 \leq j \leq p)$ are solutions of the following system of linear equations:

$$
C_{0}^{[j]} R_{j}^{\left(m_{j}-1\right)}(0)=1 \text { and } \sum_{l=0}^{s-1} C_{l}^{[j]} R_{j}^{\left(m_{j}-s+l\right)}(0)=0 \text { for } 2 \leq s \leq m_{j},
$$

where the coefficients $C_{l}^{[j]}\left(0 \leq l \leq \sum_{i=1, i \neq j} m_{i}\right)$ are given by (3.13).

As shown in Theorem 3.4 the process of elimination is simple and the technique seems to be more appropriate (from a computational point of view) for deriving the explicit formulas of the coefficients of the polynomials $R_{j}(t)(0 \leq j \leq p)$.

In the next Section we supply another process for the explicit expression of the dynamical solution and obtain the Fibonacci-Horner decomposition of $e^{t A}$.

\section{Fibonacci-Horner decomposition of $e^{t A}$ : functional process.}

4.1. Explicit formula of the dynamical solution. Let $A \in M(r ; \mathbb{C})(r \geq 2)$, whose characteristic polynomial is $P(z)=\prod_{j=1}^{p}\left(z-\lambda_{j}\right)^{m_{j}}\left(\lambda_{i} \neq \lambda_{j}\right.$ for $\left.i \neq j\right)$. Let $\varphi(t)=R_{1}(t) e^{\lambda_{1} t}+\cdots+R_{p}(t) e^{\lambda_{p}}$, where $R_{j}(t)(1 \leq j \leq s)$ is a polynomial of degree $m_{j}-1$, be the dynamical solution associated with the matrix $A$. In this subsection we give a functional method for computing explicit formulas of the polynomials $R_{j}(t)$ $(1 \leq j \leq p)$ and derive the Fibonacci-Horner decomposition of the exponential $e^{t A}$.

For reasons of clarity, let us first illustrate our method in the case $p=3$. Suppose that $P(z)=\prod_{j=1}^{3}\left(z-\lambda_{j}\right)^{m_{j}}$, where $\lambda_{i} \neq \lambda_{j}$ for $1 \leq i \neq j \leq 3$ and $m_{1} \geq 1, m_{2} \geq 1$ and $m_{3} \geq 1$. Let $\varphi(t)=R_{1}(t) e^{t \lambda_{1}}+R_{2}(t) e^{t \lambda_{2}}+R_{3}(t) e^{t \lambda_{3}}$ be the associated dynamical solution, where $R_{1}(t), R_{2}(t), R_{3}(t)$ are polynomials of degree $m_{1}-1, m_{2}-1, m_{3}-1$ (respectively). We project to determine the polynomial $R_{1}(t)$ by computing its Taylor coefficients $R_{1}^{(k)}(0)\left(0 \leq k \leq m_{1}-1\right)$. To this aim, consider the polynomial function,

$$
Q_{1}(z)=\left(z+\lambda_{1}-\lambda_{2}\right)^{m_{2}}\left(z+\lambda_{1}-\lambda_{3}\right)^{m_{3}} .
$$

A direct verification shows that expression (3.9) of $C_{s}$ is given by $C_{s}=\frac{1}{s !} \frac{d^{s} Q_{1}}{d z^{s}}(0)$. Therefore, the solution of the linear system of equations (3.10) is

$$
R_{1}^{(k)}(0)=\frac{1}{\left(m_{1}-k-1\right) !} \frac{d^{\left(m_{1}-k-1\right)}\left(\frac{1}{Q_{1}}\right)}{d z^{\left(m_{1}-k-1\right)}}(0) .
$$

To avoid confusion, in the rest of this subsection it is convenient to denote $R_{1}^{(k)}(0)$ by $\gamma_{k}^{[1]}\left(\lambda_{1}, \lambda_{2}, \lambda_{3}\right)$. A straightforward computation, using the derivation Leibniz rule, 
allows us to establish that (4.1) can be written as

$$
\gamma_{k}^{[1]}\left(\lambda_{1}, \lambda_{2}, \lambda_{3}\right)=(-1)^{m_{1}+k-1} \sum_{n_{2}+n_{3}=m_{1}-k-1} \frac{\left(\begin{array}{l}
n_{2} \\
n_{2}+m_{2}-1
\end{array}\right)\left(\begin{array}{l}
n_{3} \\
n_{3}+m_{3}-1
\end{array}\right)}{\left(\lambda_{1}-\lambda_{2}\right)^{n_{2}+m_{2}}\left(\lambda_{1}-\lambda_{3}\right)^{n_{3}+m_{3}}} .
$$

Thus the Taylor expansion of the polynomial $R_{1}(t)$ is given by

$$
R_{1}(t)=\sum_{k=0}^{m_{1}-1} \frac{\gamma_{k}^{[1]}\left(\lambda_{1}, \lambda_{2}, \lambda_{3}\right)}{k !} t^{k}
$$

where $\gamma_{k}^{[1]}\left(\lambda_{1}, \lambda_{2}, \lambda_{3}\right)$ is given by (4.2). In the same way, an identical computation implies that the polynomials $R_{2}(t)$ and $R_{3}(t)$ are given by

$$
R_{2}(t)=\sum_{k=0}^{m_{2}-1} \frac{\gamma_{k}^{[2]}\left(\lambda_{1}, \lambda_{2}, \lambda_{3}\right)}{k !} t^{k} \text { and } R_{3}(t)=\sum_{k=0}^{m_{3}-1} \frac{\gamma_{k}^{[3]}\left(\lambda_{1}, \lambda_{2}, \lambda_{3}\right)}{k !} t^{k}
$$

where $\gamma_{k}^{[2]}\left(\lambda_{1}, \lambda_{2}, \lambda_{3}\right)=\gamma_{k}^{[1]}\left(\lambda_{2}, \lambda_{1}, \lambda_{3}\right)$ and $\gamma_{k}^{[3]}\left(\lambda_{1}, \lambda_{2}, \lambda_{3}\right)=\gamma_{k}^{[1]}\left(\lambda_{3}, \lambda_{1}, \lambda_{2}\right)$. Thus we have the following proposition.

Proposition 4.1. Let $A \in M(r ; \mathbb{C})$ with characteristic polynomial $P(z)=$ $\left(z-\lambda_{1}\right)^{m_{1}}\left(z-\lambda_{2}\right)^{m_{2}}\left(z-\lambda_{3}\right)^{m_{3}}\left(m_{1}+m_{2}+m_{3}=r\right)$, where $\lambda_{i} \neq \lambda_{j}$ for $i \neq j$. Then, $e^{t A}=\varphi^{(r-1)}(t) A_{0}+\varphi^{(r-2)}(t) A_{1}+\cdots+\varphi(t) A_{r-1}$, where $\varphi(t)=R_{1}(t) e^{t \lambda_{1}}+$ $R_{2}(t) e^{t \lambda_{2}}+R_{3}(t) e^{t \lambda_{3}}$ is the associated dynamical solution such that the polynomials $R_{1}(t), R_{2}(t)$ and $R_{3}(t)$ are given by (4.3) and (4.4).

Now let $A \in M(r ; \mathbb{C})$ and suppose that its characteristic polynomial is $P(z)=$ $\prod_{j=0}^{p}\left(z-\lambda_{j}\right)^{m_{j}}$, where $\lambda_{i} \neq \lambda_{j}$ for $i \neq j$. In the aim to generalize the preceding method, let us consider the polynomial function

$$
Q_{i}(z)=\prod_{j=1, i \neq j}^{p}\left(z+\lambda_{i}-\lambda_{j}\right)^{m_{j}}
$$

Following the same method, as in the case $p=3$, we obtain,

$$
\gamma_{k}^{[i]}\left(\lambda_{1}, \cdots, \lambda_{p}\right)=\frac{1}{\left(m_{i}-k-1\right) !} \frac{d^{\left(m_{i}-k-1\right)}\left(\frac{1}{Q_{i}}\right)}{d z^{\left(m_{i}-k-1\right)}}(0) .
$$

And a long hard computation established by induction with purpose of obtaining $\frac{d^{\left(m_{i}-k-1\right)}\left(\frac{1}{Q_{i}}\right)}{d z^{\left(m_{i}-k-1\right)}}(0)$ in terms of $\lambda_{1}, \cdots, \lambda_{p}$ yields

$$
\gamma_{k}^{[i]}\left(\lambda_{1}, \cdots, \lambda_{p}\right)=(-1)^{r-m_{i}} \sum_{\sum n_{j}=m_{i}-k-1} \prod_{1 \leq j \neq i \leq p} \frac{\left(\begin{array}{l}
n_{j} \\
n_{j}+m_{j}-1
\end{array}\right)}{\left(\lambda_{j}-\lambda_{i}\right)^{n_{j}+m_{j}}}
$$

In the latter expression we suppose that we have naturally $n_{j} \leq m_{j}$. 
THEOREM 4.2. Let $A \in M(r ; \mathbb{C})(r \geq 2)$ with characteristic polynomial $P(z)=$ $\prod_{j=1}^{p}\left(z-\lambda_{j}\right)^{m_{j}}$, where $p \geq 3, m_{1}+\cdots+m_{p}=r$ and $\lambda_{i} \neq \lambda_{j}$ for $i \neq j$. Then, $e^{t A}=\varphi^{(r-1)}(t) A_{0}+\varphi^{(r-2)}(t) A_{1}+\cdots+\varphi(t) A_{r-1}$, where $\varphi(t)=\sum_{j=1}^{p} R_{j}(t) e^{\lambda_{j} t}$ is the dynamical solution, whose associated polynomials $R_{j}(t)(1 \leq j \leq p)$ are expressed as follows:

$$
R_{j}(t)=\sum_{k=0}^{m_{j}-1} \frac{\gamma_{k}^{[j]}\left(\lambda_{1}, \cdots, \lambda_{p}\right)}{k !} t^{k}
$$

where the coefficients $\gamma_{k}^{[j]}\left(\lambda_{1}, \cdots, \lambda_{p}\right)$ are given by (4.6).

For $p=3$ in (4.6), we can recover expressions for $\gamma_{k}^{[1]}\left(\lambda_{1}, \lambda_{2}, \lambda_{3}\right), \gamma_{k}^{[2]}\left(\lambda_{1}, \lambda_{2}, \lambda_{3}\right)$ and $\gamma_{k}^{[3]}\left(\lambda_{1}, \lambda_{2}, \lambda_{3}\right)$. We can also show that Proposition 4.1 is a corollary of Theorem 4.2 .

4.2. Connection with the Verde-Star's approach. As pointed out in Remark 2.3, in this subsection we turn our attention to how our approach is connected to Verde-Star's approach. First, we notice that the dynamical solution given in Theorem 4.2, can be expressed differently using Verde-Star's approach. Indeed, we can show that $R_{j}(t)=L_{k, j} 1$, where $L_{k, j}\left(1 \leq k \leq p, 0 \leq j \leq m_{k}\right)$ are the functionals associated with the polynomial $P(z)=\prod_{j=1}^{p}\left(z-\lambda_{j}\right)^{m_{j}}$, defined by $L_{k, j} f=E_{\lambda_{k}} d^{j}\left(\frac{f}{P_{k}}\right)$, where $E_{a}(f)=f(a), d^{j}=\frac{D^{j}}{j !}(D$ is ordinary operator of differentiation $)$ and $P_{k}(z)=\frac{P(z)}{\left(z-\lambda_{k}\right)^{m_{k}}}$ (see (3.2)-(3.6) of [13] for more details). More precisely, the result of Theorem 4.2 is expressed in Corollary 4.2 of [13] in terms of the functionals $L_{k, j}$.

Second, in [14] Verde-Star has arrived at the result of Theorem 4.2 using the sequence of Horner polynomials $\left\{w_{k}(z)\right\}_{k}$ associated with a given polynomial $w(z)=$ $z^{m+1}+b_{1} z^{m}+b_{2} z^{m-1}+\cdots+b_{m}$ and the function $w^{\star}(t)=(1-z t) \sum_{k \geq 0} w_{k}(z) t^{k}$. The formulation of $\frac{1}{w^{\star}(t)}$ given by Verde-Star is

$$
\frac{1}{w^{\star}(t)}=\sum_{n \geq 0} h_{n} t^{n}
$$

where $h_{n}=\sum_{j ;|j|=n} \prod_{i=0}^{p}\left(\begin{array}{c}m_{i}+j_{i} \\ j_{i}\end{array}\right) \lambda_{i}^{j}$ with $j=\left(j_{0}, j_{1}, \cdots, j_{p}\right),|j|=j_{1}+j_{2}+\cdots+j_{p}$ and $\left\{\lambda_{j}\right\}_{0 \leq j \leq p}$ are the roots of $w(z)$ of multiplicities $\left\{m_{j}\right\}_{0 \leq j \leq p}$, respectively. We infer that for $w(z)=P(z)$ the sequence $\left\{h_{n}\right\}_{n \geq 0}$ is nothing else but the $r$-generalized Fibonacci sequence $\left\{u_{n}\right\}_{n \geq 0}$ considered in Section 2 (see Expression (2.4)). By the way, we are going to digress from our aim in order to mention that this incorporation allows us to obtain another explicit expression for the sequence $\left\{u_{n}\right\}_{n \geq 0}$ in terms of $\lambda_{j}(1 \leq j \leq p)$, which will be useful in improving some results of [1]. Meanwhile, 
for $w(z)=P(z)$ the formulas (5.7) and (5.12) of [14], can help us to deduce that the coefficients of the polynomials $R_{j}(t)(1 \leq j \leq p)$ are merely the coefficients $\alpha_{j, k}$ that figure in the partial fraction decomposition of $\frac{1}{P(t)}$ given by

$$
\frac{1}{P(t)}=\sum_{j=1}^{p} \sum_{k=0}^{m_{j}-1} \alpha_{j, k} \frac{1}{\left(t-\lambda_{j}\right) .^{k}} .
$$

Moreover, following a known result, the dynamical solution $\varphi(t)$ is the divided difference with respect to the roots of the polynomial $P(z)$ of the function $e^{t z}$ (see Corollary 4.1 of [13]). Therefore, $\varphi(t)$ is the sum of the residues of the function $e^{t z} / P(z)$ at the distinct roots (see [13]). By considering this latter function $g(x, t)=e^{t x} / P(x)$ $(x \in \mathbb{R})$, a direct computation allows us to recover the result of Proposition 4.1.

5. Application to the matrix functions. Consider an analytic function $f$ on a disc $D_{K}=\{z ;|z|<K\}$ and set

$$
f(z)=\sum_{n=0}^{+\infty} \frac{f^{(n)}(0)}{n !} z^{n}
$$

Let $A \in M(r ; \mathbb{C})$ with characteristic polynomial $P_{A}(z)=z^{r}-a_{0} z^{r-1}-\cdots-a_{r-1}$ such that $K>\operatorname{Max}\left\{\left|\lambda_{j}\right| ; P_{A}\left(\lambda_{j}\right)=0\right\}$. Then, from (2.1)-(2.2) and (5.1) we derive that,

$$
f(A)=\sum_{k=0}^{r-1} \Omega_{k}(f) A_{k}, \quad \text { where } \Omega_{k}(f)=\sum_{n=0}^{+\infty} u_{n-k} \frac{f^{(n)}(0)}{n !} .
$$

Recall that in Proposition 2.1, we had considered the (formal) analytic function (2.6) defined by

$$
\varphi(t)=\sum_{n=0}^{+\infty} u_{n} \frac{t^{n+r-1}}{(n+r-1) !} .
$$

The above expression shows that $u_{n-r+1}=\varphi^{(n)}(0)$. On the other hand, (3.1) shows that $\varphi^{(n)}(t)=\sum_{i=1}^{p} \sum_{s=n-m_{i}+1}^{n}\left(\begin{array}{l}s \\ n\end{array}\right) \lambda_{i}^{s} R_{i}^{(n-s)}(t)$. Hence, using (5.2) we have the following proposition.

Proposition 5.1. Under the preceding hypotheses, we have $f(A)=\sum_{k=0}^{r-1} \Omega_{k}(f) A_{k}$, where

$$
\Omega_{k}(f)=\sum_{i=1}^{p}\left[\sum_{n=k}^{+\infty} \Gamma_{i, n}\left(\lambda_{1}, \cdots, \lambda_{p}\right) \frac{f^{(n)}(0)}{n !}\right]
$$


with

$$
\Gamma_{i, n}\left(\lambda_{1}, \cdots, \lambda_{p}\right)=\sum_{s=n-k+r-m_{i}}^{n-k+r-1}\left(\begin{array}{l}
s \\
n-k+r-1
\end{array}\right) \lambda_{i}^{s} Y_{n-s+r-k-1}^{<i>}\left(\lambda_{1}, \cdots, \lambda_{p}\right),
$$

where we have set

$$
Y_{n}^{<i>}\left(\lambda_{1}, \cdots, \lambda_{p}\right)=R_{i}^{(n)}(0)
$$

To the best of our knowledge, expressions (5.2)-(5.3) of $f(A)$ are new in the literature. Note that a formula analogous to (5.2) also appears in [14]. We would like to note that it is an interesting issue to illustrate the deep relationship between our development and that of Verde-Star, even though the two approaches are based on different techniques.

6. Examples. In the two first examples we illustrate the applicability of Theorem 4.2. The last example concerns the practical use of Proposition 5.1 on a classical matrix function.

Example 1. Suppose that $A \in M(r ; \mathbb{C})(r \geq 2)$ has characteristic polynomial with simple roots, that is, $P(z)=\prod_{j=1}^{r}\left(z-\lambda_{j}\right)$, such that $\lambda_{i} \neq \lambda_{i}(i \neq j)$. Then, the dynamical solution associated with $A$ is given by $\varphi(t)=R_{1} e^{\lambda_{1} t}+\cdots+R_{r} e^{\lambda_{r} t}$, where $R_{i}(t)=R_{i}$ is a constant polynomial for every $i(1 \leq i \leq r)$. Application of (4.6) allows us to derive that

$$
R_{i}=\gamma_{0}^{[i]}=(-1)^{r-1} \frac{1}{\prod_{j \neq i}\left(\lambda_{j}-\lambda_{i}\right)}=\frac{1}{\prod_{j \neq i}\left(\lambda_{i}-\lambda_{j}\right)}=\frac{1}{P^{\prime}\left(\lambda_{i}\right)} .
$$

Therefore, the dynamical solution associated with matrix $A$ is

$$
\varphi(t)=\sum_{i=1}^{r} \frac{e^{t \lambda_{i}}}{P^{\prime}\left(\lambda_{i}\right)}
$$

Expression (6.1) is well-known in the literature; see, e.g., [2, 9, 13]. Hence, the decomposition of $e^{t A}$ in the Fibonacci-Horner basis can be obtained easily.

Example 2. Suppose that $A \in M(3 ; \mathbb{C})$ has characteristic polynomial $P(z)=$ $\left(z-\lambda_{1}\right)^{2}\left(z-\lambda_{2}\right)$, where $\lambda_{1} \neq \lambda_{2}$. Then the dynamical solution associated with $A$ is given by $\varphi(t)=R_{1}(t) e^{\lambda_{1} t}+R_{2} e^{\lambda_{2} t}$, where $R_{1}(t)$ is a polynomial of degree 1 and $R_{2}$ is a constant polynomial. More precisely, it is easy to show that $R_{1}(t)=\gamma_{0}^{[1]}+\gamma_{1}^{[1]} t$ and $R_{2}(t)=\gamma_{0}^{[2]} \in \mathbb{C}$. Application of (4.6) shows that

$$
\gamma_{0}^{[1]}=-\frac{1}{\left(\lambda_{1}-\lambda_{2}\right)^{2}}, \gamma_{1}^{[1]}=\frac{1}{\left(\lambda_{1}-\lambda_{2}\right)} \text { and } \gamma_{0}^{[2]}=\frac{1}{\left(\lambda_{1}-\lambda_{2}\right)^{2}} .
$$

The dynamical solution associated with the matrix $A$ is given by

$$
\varphi(t)=\left[-\frac{1}{\left(\lambda_{1}-\lambda_{2}\right)^{2}}+\frac{t}{\left(\lambda_{1}-\lambda_{2}\right)}\right] e^{\lambda_{1} t}+\frac{1}{\left(\lambda_{1}-\lambda_{2}\right)^{2}} e^{\lambda_{2} t} .
$$


Thus, the decomposition of $e^{t A}$ in the Fibonacci-Horner basis can be derived easily.

Example 3. As in Example 2, let $A \in M(3 ; \mathbb{C})$ whose characteristic polynomial is $P(z)=\left(z-\lambda_{1}\right)^{2}\left(z-\lambda_{2}\right)$ with $\lambda_{1} \neq \lambda_{2}$. Consider the function $f(z)=\log (1+z)$. If we consider the analytic expression $f(z)=\sum_{n=1}^{+\infty}(-1)^{n+1} \frac{z^{n}}{n}$, a straightforward application of Proposition 5.1 implies that $f(A)=\Omega_{0}(f) A_{0}+\Omega_{1}(f) A_{1}$, where

$$
\Omega_{0}(f)=\sum_{n=1}^{+\infty}\left[\frac{\lambda_{2}^{n+2}-\lambda_{1}^{n+2}}{\left(\lambda_{2}-\lambda_{1}\right)^{2}}+(n+2) \frac{\lambda_{1}^{n+1}}{\lambda_{1}-\lambda_{2}}\right] \frac{(-1)^{n-1}}{n}
$$

and

$$
\Omega_{1}(f)=\sum_{n=1}^{+\infty}\left[\frac{\lambda_{2}^{n+1}-\lambda_{1}^{n+1}}{\left(\lambda_{2}-\lambda_{1}\right)^{2}}+(n+1) \frac{\lambda_{1}^{n}}{\lambda_{1}-\lambda_{2}}\right] \frac{(-1)^{n-1}}{n} .
$$

Acknowledgment. We would like to express deep gratitude to a referee for valuable suggestions that improved a large part of this paper, especially Section 4 . References [5] and [14] were also pointed out by the referee. We thank Professor L. Verde-Star for his kindness in sending us some of his papers.

\section{REFERENCES}

[1] R. Ben Taher and M. Rachidi. Linear recurrence relations in the algebra of matrices and applications. Linear Algebra and its Applications, 330:15-24, 2001.

[2] R. Ben Taher and M. Rachidi. Some explicit formulas for the polynomial decomposition of the matrix exponential and applications. Linear Algebra and its Applications, 350(1-3):171-184, 2002.

[3] R. Ben Taher and M. Rachidi. Application of the $\epsilon$-algorithm to the ratios of $r$-generalized Fibonacci sequences. Fibonacci Quarterly, 39(1):22-26, 2001.

[4] F.R. Gantmacher. Theory of matrices. Chelsea Publishing Company, New York, 1959.

[5] P. Henrici. Applied and Computational Complex Analysis, Vol. 1. J. Wiley, New York, 1974.

[6] I.I. Kolodner. On $\exp (t A)$ with $A$ satisfying a polynomial. Journal of Mathematical Analysis and Applications, 52:514-524, 1975.

[7] I.E. Leonardo. The matrix exponential. SIAM Review, 38(3):507-512, 1996.

[8] C. Levesque. On $m$-th order linear recurrences. Fibonacci Quarterly, 23(4):290-295, 1985.

[9] C. Moler and C. Van Loan. Nineteen dubious ways to compute the exponential of matrix. SIAM Review, 20(4):801-836, 1978.

[10] M. Mouline and M. Rachidi. Application of Markov Chains properties to r-Generalized Fibonacci Sequences. Fibonacci Quarterly, 37:34-38, 1999.

[11] M. Mouline and M. Rachidi. Suites de Fibonacci généralisées, Théorème de Cayley-Hamilton et chaines de Markov. Rendiconti Sem. Mat. di Messina, No. 4 Serie II, T. XIX (1996/97), pp. 107-115.

[12] E.J. Putzer. Avoiding the Jordan canonical form in the discussion of linear systems with constant coefficients. American Mathematical Monthly, 73:2-7, 1966.

[13] L. Verde-Star. Operator identities and the solution of linear matrix difference and differential equations. Studies in Applied Mathematics, 91:153-177, 1994.

[14] L. Verde-Star. Functions of matrices. Linear Algebra and its Applications, 406:285-300, 2005. 